Ezio Marchi

IMA Preprint Series \# 2200

(April 2008)

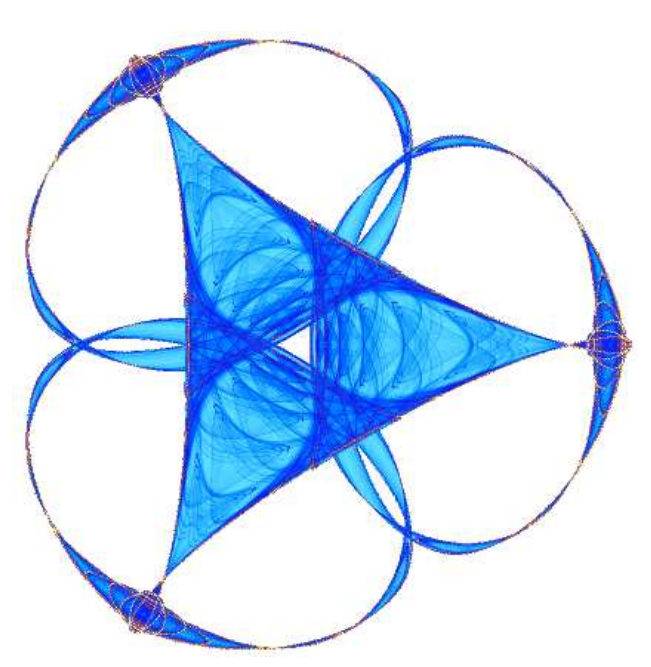

INSTITUTE FOR MATHEMATICS AND ITS APPLICATIONS UNIVERSITY OF MINNESOTA 400 Lind Hall 207 Church Street S.E. Minneapolis, Minnesota 55455-0436

Phone: 612-624-6066 Fax: 612-626-7370

URL: http://www.ima.umn.edu 


\title{
E-points for diagonal games III
}

by

Ezio Marchi ${ }^{* * *}$

\begin{abstract}
In this paper we study and compute $E$-points in an explicit way for a special kind of games with $3 \mathrm{k}+1,4 \mathrm{k}+3$ and $\mathrm{sk}+1$ with $1 \leq \mathrm{s} \leq \mathrm{k}$.
\end{abstract}

Key words: Equilibrium points, $E$-points, non-cooperative games.

\footnotetext{
*) Founder and First Director of the Instituto de Matemática Aplicada, San Luis, CONICET and Universidad Nacional de San Luis, San Luis, Argentina. Visiting the University of Barcelona, Spain. e-mail: emarchi@sinectis.com.ar

${ }^{* * *}$ This paper has been written in the Department of Applied Mathematics and Analysis, University of Barcelona, Barcelona, Spain, during a visit supported by the Dirección General de Investigaciones Científicas y Técnicas (DGIGYT) of Spain. The author acknowledges the hospitality of the Department.
} 


\section{Introduction}

In two previous papers Marchi (2004) we have begun to compute in an explicit way $E$-points for diagonal games. The games presented there are simple. Here in this paper we continue the computations of $E$-points in diagonal games for more difficult cases.

With notation of Marchi (2004) we have that the expected functions $E_{i}$ for player $\mathrm{i} \in \mathrm{N}=\{1, \ldots, \mathrm{n}\}$ and $\mathrm{d}(\mathrm{i}) \subset \mathrm{N}$ the set the friends players of player $\mathrm{i} \in \mathrm{N}$ determines all the structure of the game $(F, E)$. We remind that an $E$-point is a point $\overline{\mathrm{x}}=\left(\overline{\mathrm{x}}_{1}, \ldots, \overline{\mathrm{x}}_{\mathrm{n}}\right)$ such that

$$
\mathrm{E}_{\mathrm{i}}(\overline{\mathrm{x}}) \geq \mathrm{E}_{\mathrm{i}}\left(\mathrm{x}_{\mathrm{d}(\mathrm{i})}, \overline{\mathrm{x}}_{\mathrm{N}-\mathrm{d}(\mathrm{i})}\right) \quad \forall \mathrm{i} \forall \mathrm{x}_{\mathrm{d}(\mathrm{i})}
$$

In Marchi (2004) we have proved the following result:

Preposition: $\overline{\mathrm{x}}$ is an $E$-point if and only if

$$
\begin{array}{cc}
\lambda_{\mathrm{i}}-\mathrm{E}_{\mathrm{i}}\left(\sigma_{\mathrm{d}(\mathrm{i})}, \overline{\mathrm{x}}_{\mathrm{N}-\mathrm{d}(\mathrm{i})}\right)=0 & \forall \sigma_{\mathrm{d}(\mathrm{i})} \in \prod_{\mathrm{j} \in \mathrm{d}(\mathrm{i})} \mathrm{S}\left(\overline{\mathrm{x}}_{\mathrm{j}}\right) \\
\lambda_{\mathrm{i}}-\mathrm{E}_{\mathrm{i}}\left(\sigma_{\mathrm{d}(\mathrm{i})}, \overline{\mathrm{x}}_{\mathrm{N}-\mathrm{d}(\mathrm{i})}\right) \geq 0 & \forall \sigma_{\mathrm{d}(\mathrm{i})} \notin \prod_{\mathrm{j} \in \mathrm{d}(\mathrm{i})} \mathrm{S}\left(\overline{\mathrm{x}}_{\mathrm{j}}\right) \\
\sum_{\sigma_{\mathrm{i}} \in \Sigma_{\mathrm{i}}} \overline{\mathrm{x}}_{\mathrm{i}}\left(\sigma_{\mathrm{i}}\right)=1 & \forall \mathrm{i} \\
\overline{\mathrm{x}}_{\mathrm{i}}\left(\sigma_{\mathrm{i}}\right) \geq 0 \quad \forall \mathrm{i} \quad \forall \sigma_{\mathrm{i}} \in \Sigma_{\mathrm{i}} .
\end{array}
$$

where $S\left(\bar{x}_{j}\right)$ denotes the support of the mixed strategy $\bar{x}_{j}$.

We consider in this note that all the players have the same cardinality for their pure strategy set: $\mathrm{m}=\left|\Sigma_{\mathrm{i}}\right|$.

In the next section we study two games namely one with $4 \mathrm{k}+1$ and $4 \mathrm{k}+3$ players respectively and in the third section a general game with $\mathrm{sk}+1,1 \leq \mathrm{s} \leq \mathrm{k}$. All of them have a similar structure function. But they are more complicated that those presented in Marchi (2004). 


\section{General games with $4 k+1$ and $4 k+3$ players with $k \geq 1$}

Here in this section we are going to compute $E$-points for general diagonal games having respectively $4 \mathrm{k}+1$ and $4 \mathrm{k}+3$ players.

Consider the game $\Gamma$ with $n$-players with the structure function given by $\mathrm{d}(\mathrm{i})=\mathrm{N}-\{\mathrm{i}+2, \mathrm{i}+3, \mathrm{i}+4, \mathrm{i}+5\} \bmod 3 \mathrm{k}+1$ with $1 \leq \mathrm{k}$. Therefore the payoff function of player $\mathrm{i} \in \mathrm{N}$ for the diagonal game $(\Gamma, E)$ is given by

$$
\mathrm{A}_{\mathrm{i}}\left(\sigma_{\mathrm{i}}, \ldots, \sigma_{4 \mathrm{k}+1}\right)=\mathrm{a}_{\mathrm{i}}\left(\sigma_{\mathrm{i}}\right) \delta\left(\sigma_{\mathrm{i}}, \sigma_{\mathrm{i}+2}, \sigma_{\mathrm{i}+3}, \sigma_{\mathrm{i}+4}, \sigma_{\mathrm{i}+5}\right), \quad \mathrm{a}_{\mathrm{i}}\left(\sigma_{\mathrm{i}}\right)>0,
$$

where

$$
\delta\left(\sigma_{i}, \sigma_{i+2}, \sigma_{i+3}, \sigma_{i+4}, \sigma_{i+5}\right)=\delta\left(\sigma_{i}, \sigma_{i+2}\right) \delta\left(\sigma_{i+2}, \sigma_{i+3}\right) \delta\left(\sigma_{i+3}, \sigma_{i+4}\right) \delta\left(\sigma_{i+4}, \sigma_{i+5}\right),
$$

with Krorecker's delta $\delta$ 's .

A completely mixed strategy is a mixed strategy $\mathrm{x}=\left(\mathrm{x}_{1}, \ldots, \mathrm{x}_{4 \mathrm{k}+1}\right)$ such that $\forall \mathrm{i} \forall \sigma_{\mathrm{i}} \in \Sigma_{\mathrm{i}}: \mathrm{x}_{\mathrm{i}}\left(\sigma_{\mathrm{i}}\right)>0$.

We wish to compute the completely mixed $E$-points for our game. By the preposition we have to solve

$$
\lambda_{\mathrm{i}}-\mathrm{a}_{\mathrm{i}}(\sigma) \mathrm{x}_{\mathrm{i}+2}(\sigma) \mathrm{x}_{\mathrm{i}+3}(\sigma) \mathrm{x}_{\mathrm{i}+4}(\sigma) \mathrm{x}_{\mathrm{i}+5}(\sigma)=0 \quad \forall \mathrm{i} \quad \bmod 4 \mathrm{k}+1 \quad \forall \sigma: 1, \ldots, \mathrm{m}
$$

For reasons of simplicity we drop in the notation the independent variable, in other words $\mathrm{a}_{\mathrm{i}}=\mathrm{a}_{\mathrm{i}}(\sigma)$ and $\mathrm{x}_{\mathrm{i}}(\sigma)=\mathrm{x}_{\mathrm{i}}$. Calling $\bar{\mu}_{\mathrm{i}}=\bar{\mu}_{\mathrm{i}}(\sigma)=\lambda_{\mathrm{i}} / \mathrm{a}_{\mathrm{i}}$ and $\bar{\mu}_{\mathrm{i}}=\mu_{\mathrm{i}+2}$ we have

$$
\mu_{\mathrm{i}+2}-\mathrm{x}_{\mathrm{i}+2} \mathrm{x}_{\mathrm{i}+3} \mathrm{x}_{\mathrm{i}+4} \mathrm{x}_{\mathrm{i}+5}=0 \quad \bmod 4 \mathrm{k}+1 .
$$

From two consecutives equations we have

$$
\mathrm{x}_{\mathrm{i}+6}=\frac{\mu_{\mathrm{i}+3}}{\mu_{\mathrm{i}+2}} \mathrm{x}_{\mathrm{i}+2}
$$

and recursively

$$
x_{i+4}=\prod_{s=0}^{r} S_{i+1-4 s} x_{i-4 r}
$$

with $S_{i+1}=\frac{\mu_{i+1}}{\mu_{i}}$. From now on if it not necessary we assume implicitly that all our equations are $\bmod 4 \mathrm{k}+1$.

Consider a $1 \leq \mathrm{p} \leq \mathrm{k}$. Then the corresponding equation is 


$$
\mu_{4 \mathrm{p}+1}-\mathrm{x}_{4 \mathrm{p}+1} \mathrm{x}_{4 \mathrm{p}+2} \mathrm{x}_{4 \mathrm{p}+3} \mathrm{x}_{4 \mathrm{p}+4}=0
$$

Take in (4) $\mathrm{i}=4(\mathrm{p}+\mathrm{k})+1$ and $\mathrm{r}=\mathrm{k}$ then we have

$$
\mathrm{x}_{4 \mathrm{p}+4}=\prod_{\mathrm{s}=0}^{\mathrm{k}} \mathrm{S}_{4(\mathrm{p}+\mathrm{k})+2-4 \mathrm{~s}} \mathrm{x}_{4 \mathrm{p}+1}
$$

On the other hand if $\mathrm{i}=4(\mathrm{p}+2 \mathrm{k})+1$ and $\mathrm{r}=2 \mathrm{k}$ it holds true

$$
\mathrm{x}_{4 \mathrm{p}+3}=\prod_{\mathrm{s}=0}^{2 \mathrm{k}} \mathrm{S}_{4(\mathrm{p}+\mathrm{k})+2-4 \mathrm{~s}} \mathrm{x}_{4 \mathrm{p}+1}
$$

Finally if $i=4(p+3 k)+1$ and $r=3 k$ it appears

$$
\mathrm{x}_{4 \mathrm{p}+2}=\prod_{\mathrm{s}=0}^{3 \mathrm{k}} \mathrm{S}_{4(\mathrm{p}+3 \mathrm{k})+2-4 \mathrm{~s}} \mathrm{x}_{4 \mathrm{p}+1}
$$

Replacing (6), (7) and (8) in (5) we derive

$$
\mu_{4 \mathrm{p}+1}-\mathrm{x}_{4 \mathrm{p}+1}^{4} \prod_{\mathrm{s}=0}^{\mathrm{k}} \mathrm{S}_{4(\mathrm{p}+\mathrm{k})+2-4 \mathrm{~s}} \prod_{\mathrm{s}=0}^{2 \mathrm{k}} \mathrm{S}_{4(\mathrm{p}+3 \mathrm{k})+2-4 \mathrm{~s}} \prod_{\mathrm{s}=0}^{3 \mathrm{k}} \mathrm{S}_{4(\mathrm{p}+3 \mathrm{k})+2-4 \mathrm{~s}}=0
$$

In a similar way for $4 p+2,4 p+3$ and $4 p+4$ :

$$
\begin{aligned}
& \mu_{4 \mathrm{p}+2}-\mathrm{x}_{4 \mathrm{p}+2}^{4} \prod_{\mathrm{s}=0}^{\mathrm{k}} \mathrm{S}_{4(\mathrm{p}+\mathrm{k})+3-4 \mathrm{~s}} \prod_{\mathrm{s}=0}^{2 \mathrm{k}} \mathrm{S}_{4(\mathrm{p}+2 \mathrm{k})+3-4 \mathrm{~s}} \prod_{\mathrm{s}=0}^{3 \mathrm{k}} \mathrm{S}_{4(\mathrm{p}+3 \mathrm{k})+3-4 \mathrm{~s}}=0 \\
& \mu_{4 \mathrm{p}+3}-\mathrm{x}_{4 \mathrm{p}+3}^{4} \prod_{\mathrm{s}=0}^{\mathrm{k}} \mathrm{S}_{4(\mathrm{p}+\mathrm{k})+4-4 \mathrm{~s}} \prod_{\mathrm{s}=0}^{2 \mathrm{k}} \mathrm{S}_{4(\mathrm{p}+2 \mathrm{k})+4-4 \mathrm{~s}} \prod_{\mathrm{s}=0}^{3 \mathrm{k}} \mathrm{S}_{4(\mathrm{p}+3 \mathrm{k})+4-4 \mathrm{~s}}=0
\end{aligned}
$$

and

$$
\mu_{4 \mathrm{p}+4}-\mathrm{x}_{4 \mathrm{p}+4}^{4} \prod_{\mathrm{s}=0}^{\mathrm{k}} \mathrm{S}_{4(\mathrm{p}+\mathrm{k})+5-4 \mathrm{~s}} \prod_{\mathrm{s}=0}^{2 \mathrm{k}} \mathrm{S}_{4(\mathrm{p}+2 \mathrm{k})+5-4 \mathrm{~s}} \prod_{\mathrm{s}=0}^{3 \mathrm{k}} \mathrm{S}_{4(\mathrm{p}+3 \mathrm{k})+5-4 \mathrm{~s}}=0
$$

Making use of the equation $\sum_{\sigma_{i}} x_{i}\left(\sigma_{i}\right)=1$ then we have from (9)

$$
\lambda_{4 \mathrm{p}-1} \frac{\prod_{\mathrm{s}=0}^{\mathrm{k}} \lambda_{4(\mathrm{p}+\mathrm{k})-1-4 \mathrm{~s}} \prod_{\mathrm{s}=0}^{2 \mathrm{k}} \lambda_{4(\mathrm{p}+2 \mathrm{k})-1-4 \mathrm{~s}} \prod_{\mathrm{s}+0}^{3 \mathrm{k}} \lambda_{4(\mathrm{p}+3 \mathrm{k})-1-4 \mathrm{~s}}}{\prod_{\mathrm{s}=0}^{\mathrm{k}} \lambda_{4(\mathrm{p}+\mathrm{k})-4 \mathrm{~s}} \prod_{\mathrm{s}=0}^{2 \mathrm{k}} \lambda_{4(\mathrm{p}+2 \mathrm{k})-4 \mathrm{~s}} \prod_{\mathrm{s}=0}^{3 \mathrm{k}} \lambda_{4(\mathrm{p}+3 \mathrm{k})-4 \mathrm{~s}}}=\mathrm{b}_{4 \mathrm{p}+1}
$$

where

$$
\begin{aligned}
& \mathrm{b}_{4 \mathrm{p}+1} \\
& =\left(\sum_{\sigma}\left(\frac{1}{\mathrm{a}_{4 \mathrm{p}+1}(\sigma)} \frac{\prod_{\mathrm{s}=0}^{\mathrm{k}} \mathrm{a}_{4(\mathrm{p}+\mathrm{k})-4 \mathrm{~s}}(\sigma) \prod_{\mathrm{s}=0}^{2 \mathrm{k}} \mathrm{a}_{4(\mathrm{p}+2 \mathrm{k})-4 \mathrm{~s}}(\sigma) \prod_{\mathrm{s}+0}^{3 \mathrm{k}} \mathrm{a}_{4(\mathrm{p}+3 \mathrm{k})-4 \mathrm{~s}}(\sigma)}{\prod_{\mathrm{s}=0}^{\mathrm{k}} \mathrm{a}_{4(\mathrm{p}+\mathrm{k})-1-4 \mathrm{~s}}(\sigma) \prod_{\mathrm{s}=0}^{2 \mathrm{k}} \mathrm{a}_{4(\mathrm{p}+2 \mathrm{k})-1-4 \mathrm{~s}}(\sigma) \prod_{\mathrm{s}+0}^{3 \mathrm{k}} \mathrm{a}_{4(\mathrm{p}+3 \mathrm{k})-1-4 \mathrm{~s}}(\sigma)}\right)^{4}\right)^{1 / 4}
\end{aligned}
$$


Similarly for $4 p+2,4 p+3$ and $4 p+4$

$$
\begin{gathered}
\lambda_{4 \mathrm{p}} \frac{\prod_{\mathrm{s}=0}^{\mathrm{k}} \lambda_{4(\mathrm{p}+\mathrm{k})-4 \mathrm{~s}} \prod_{\mathrm{s}=0}^{2 \mathrm{k}} \lambda_{4(\mathrm{p}+2 \mathrm{k})-4 \mathrm{~s}} \prod_{\mathrm{s}+0}^{3 \mathrm{k}} \lambda_{4(\mathrm{p}+3 \mathrm{k})-4 \mathrm{~s}}}{\prod_{\mathrm{s}=0}^{\mathrm{k}} \lambda_{4(\mathrm{p}+\mathrm{k})+1-4 \mathrm{~s}} \prod_{\mathrm{s}=0}^{2 \mathrm{k}} \lambda_{4(\mathrm{p}+2 \mathrm{k})+1-4 \mathrm{~s}} \prod_{\mathrm{s}=0}^{3 \mathrm{k}} \lambda_{4(\mathrm{p}+3 \mathrm{k})+1-4 \mathrm{~s}}}=\mathrm{b}_{4 \mathrm{p}+2} \\
\lambda_{4 \mathrm{p}+1} \frac{\prod_{\mathrm{s}=0}^{\mathrm{k}} \lambda_{4(\mathrm{p}+\mathrm{k})+1-4 \mathrm{~s}} \prod_{\mathrm{s}=0}^{2 \mathrm{k}} \lambda_{4(\mathrm{p}+2 \mathrm{k})+1-4 \mathrm{~s}} \prod_{\mathrm{s}=0}^{3 \mathrm{k}} \lambda_{4(\mathrm{p}+3 \mathrm{k})+1-4 \mathrm{~s}}}{\prod_{\mathrm{s}=0}^{\mathrm{k}} \lambda_{4(\mathrm{p}+\mathrm{k})+2-4 \mathrm{~s}} \prod_{\mathrm{s}=0}^{2 \mathrm{k}} \lambda_{4(\mathrm{p}+2 \mathrm{k})+2-4 \mathrm{~s}} \prod_{\mathrm{s}=0}^{3 \mathrm{k}} \lambda_{4(\mathrm{p}+3 \mathrm{k})+2-4 \mathrm{~s}}}=\mathrm{b}_{4 \mathrm{p}+3} \\
\lambda_{4 \mathrm{p}+2} \frac{\prod_{\mathrm{s}=0}^{\mathrm{k}} \lambda_{4(\mathrm{p}+\mathrm{k})+2-4 \mathrm{~s}} \prod_{\mathrm{s}=0}^{2 \mathrm{k}} \lambda_{4(\mathrm{p}+2 \mathrm{k})+2-4 \mathrm{~s}} \prod_{\mathrm{s}+0}^{\mathrm{k}} \lambda_{4(\mathrm{p}+3 \mathrm{k})+2-4 \mathrm{~s}}}{\prod_{\mathrm{s}=0}^{\mathrm{k}} \lambda_{4(\mathrm{p}+\mathrm{k})+3-4 \mathrm{~s}} \prod_{\mathrm{s}=0}^{2 \mathrm{k}} \lambda_{4(\mathrm{p}+2 \mathrm{k})+3-4 \mathrm{~s}} \prod_{\mathrm{s}=0}^{3 \mathrm{k}} \lambda_{4(\mathrm{p}+3 \mathrm{k})+3-4 \mathrm{~s}}}=\mathrm{b}_{4 \mathrm{p}+4}
\end{gathered}
$$

where the expressions of $b_{4 p+2}, b_{4 p+3}$ and $b_{4 p+4}$ are analogous to that of $b_{4 p+1}$ in (14).

Now multiplying the four amounts it turns out

$$
\begin{gathered}
\mathrm{b}_{4 \mathrm{p}+1} \mathrm{~b}_{4 \mathrm{p}+2} \mathrm{~b}_{4 \mathrm{p}+3} \mathrm{~b}_{4 \mathrm{p}+4}=\lambda_{4 \mathrm{p}-1} \lambda_{4 \mathrm{p}} \lambda_{4 \mathrm{p}+1} \lambda_{4 \mathrm{p}+2} . \\
\cdot \frac{\lambda_{4(\mathrm{p}+\mathrm{k})-1} \lambda_{4(\mathrm{p}+\mathrm{k})-5} \cdots \lambda_{4 \mathrm{p}+3} \lambda_{4 \mathrm{p}-1}}{\lambda_{4(\mathrm{p}+\mathrm{k})+3} \lambda_{4(\mathrm{p}+\mathrm{k})-1} \cdots \lambda_{4 \mathrm{p}+3}} \\
\cdot \frac{\lambda_{4(\mathrm{p}+2 \mathrm{k})-1} \lambda_{4(\mathrm{p}+2 \mathrm{k})-5} \cdots \lambda_{4 \mathrm{p}-1}}{\lambda_{4(\mathrm{p}+2 \mathrm{k})+3} \lambda_{4(\mathrm{p}+2 \mathrm{k})-1} \cdots \lambda_{4 \mathrm{p}+3}} \\
\cdot \frac{\lambda_{4(\mathrm{p}+3 \mathrm{k})-1} \lambda_{4(\mathrm{p}+3 \mathrm{k})-5} \cdots \lambda_{4 \mathrm{p}-1}}{\lambda_{4(\mathrm{p}+3 \mathrm{k})+3} \lambda_{4(\mathrm{p}+3 \mathrm{k})-1} \cdots \lambda_{4 \mathrm{p}+3}} \\
=\lambda_{4 \mathrm{p}-1}^{4} \frac{\lambda_{4 \mathrm{p}} \lambda_{4 \mathrm{p}+1} \lambda_{4 \mathrm{p}+2}}{\lambda_{4(\mathrm{p}+\mathrm{k})+3} \lambda_{4(\mathrm{p}+2 \mathrm{k})+3} \lambda_{4(\mathrm{p}+3 \mathrm{k})+3}}=\lambda_{4(\mathrm{p}-1)}^{4}
\end{gathered}
$$

But since

$$
\begin{aligned}
& 4 p+2+4 k+1=4(p+k)+3 \\
& 4 p+1+8 k+2=4(p+2 k)+3 \\
& 4 p+12 k+3=4(p+3 k)+3
\end{aligned}
$$

the last equality of (18) holds. Therefore

$$
\lambda_{4 \mathrm{p}-1}=\left(\mathrm{b}_{4 \mathrm{p}+1} \mathrm{~b}_{4 \mathrm{p}+2} \mathrm{~b}_{4 \mathrm{p}+3} \mathrm{~b}_{4 \mathrm{p}+4}\right)^{1 / 4}
$$

Thus, in a similar way it is possible to obtain

$$
\lambda_{i}=\left(b_{i+2} b_{i+3} b_{i+4} b_{i+5}\right)^{1 / 4}
$$

replacing in (10), (11), (12) the derivation of $\mathrm{x}_{\mathrm{i}}$ is immediate. Thus we have computed the only one $E$-point completely mixed for this game. 
Next we are going to study the same diagonal game but with $4 \mathrm{k}+3$ players. For our task it facilitates the operation to write the stripes

$$
\begin{array}{lrll}
1 \ldots \ldots 4 \mathrm{k}+1 & 4 \mathrm{k}+5 \equiv 2 \ldots \ldots 8 \mathrm{k}+1 & 8 \mathrm{k}+5 & 8 \mathrm{k}+9 \equiv 3 \ldots \\
2 \ldots . .4 \mathrm{k}+2 & 4 \mathrm{k}+6 \equiv 3 \ldots \ldots 8 \mathrm{k}+2 & \underline{8 \mathrm{k}+6} & 8 \mathrm{k}+10 \equiv 4 \ldots \\
3 \ldots \ldots .4 \mathrm{k}+3 & 4 \mathrm{k}+7 \equiv 4 \ldots \ldots 8 \mathrm{k}+3 & 8 \mathrm{k}+7 \equiv 1 & \ldots \ldots \\
4 \ldots \ldots 4 \mathrm{k}+4 \equiv 1 & \ldots \ldots 8 \mathrm{k}+4 & 8 \mathrm{k}+8 \equiv 2 & \ldots \ldots .
\end{array}
$$

$$
\begin{array}{lr}
\ldots \underline{12 k+9} & 12 k+13 \equiv 4 \ldots \ldots 16 k+13 \equiv 1 \\
\ldots 12 k+10 \equiv 1 & \ldots \ldots 16 k+14 \equiv 2 \\
\ldots 12 k+11 \equiv 2 & \ldots \ldots 16 k+15 \equiv 3 \\
\ldots 12 k+12 \equiv 3 & \ldots \ldots .16 k+16 \equiv 4
\end{array}
$$

in order to realize how the number formation $\bmod 4 \mathrm{k}+3$ arranges.

Using (4) with $\mathrm{i}=4(\mathrm{p}+\mathrm{k})+1$ and $\mathrm{r}=\mathrm{k}$ we obtain

$$
\mathrm{x}_{3 \mathrm{p}+2}=\prod_{\mathrm{s}=0}^{\mathrm{k}} \mathrm{S}_{4(\mathrm{p}+\mathrm{k})+2-4 \mathrm{~s}} \mathrm{x}_{4 \mathrm{p}+1}
$$

on the other hand with $\mathrm{i}=4(\mathrm{p}+2 \mathrm{k})+5$ and $\mathrm{r}=2 \mathrm{k}+1$ it holds

$$
\mathrm{x}_{3 \mathrm{p}+3}=\prod_{\mathrm{s}=0}^{2 \mathrm{k}+1} \mathrm{~S}_{4(\mathrm{p}+2 \mathrm{k})+6-4 \mathrm{~s}} \mathrm{x}_{4 \mathrm{p}+1}
$$

changing the corresponding $i$ to $i=4(p+3 k)+9$ and $r=3 k+2$ it follows

$$
\mathrm{x}_{4 \mathrm{p}+4}=\prod_{\mathrm{s}=0}^{3 \mathrm{k}+2} \mathrm{~S}_{4(\mathrm{p}+3 \mathrm{k})+10-4 \mathrm{~s}} \mathrm{x}_{4 \mathrm{p}+1}
$$

replacing the amounts in the equation (5) then we get

$$
\mu_{3 p+1}-x_{3 p+1}^{4} \prod_{s=0}^{k} S_{4(p+k)+2-4 s} \prod_{s=0}^{2 k+1} S_{4(p+2 k)+6-4 s} \prod_{s=0}^{3 k+2} S_{4(p+3 k)+10-4 s}=0
$$

Performing the same operations in the corresponding equations it is easy to obtain

$$
\begin{aligned}
& \mu_{4 \mathrm{p}+2}-\mathrm{x}_{3 \mathrm{p}+2}^{4} \prod_{\mathrm{s}=0}^{\mathrm{k}} \mathrm{S}_{4(\mathrm{p}+\mathrm{k})+3-4 \mathrm{~s}} \prod_{\mathrm{s}=0}^{2 \mathrm{k}+1} \mathrm{~S}_{4(\mathrm{p}+2 \mathrm{k})+7-4 \mathrm{~s}} \prod_{\mathrm{s}=0}^{3 \mathrm{k}+2} \mathrm{~S}_{4(\mathrm{p}+3 \mathrm{k})+11-4 \mathrm{~s}}=0 \\
& \mu_{4 \mathrm{p}+3}-\mathrm{x}_{3 \mathrm{p}+3}^{4} \prod_{\mathrm{s}=0}^{\mathrm{k}} \mathrm{S}_{4(\mathrm{p}+\mathrm{k})+4-4 \mathrm{~s}} \prod_{\mathrm{s}=0}^{2 \mathrm{k}+1} \mathrm{~S}_{4(\mathrm{p}+2 \mathrm{k})+8-4 \mathrm{~s}} \prod_{\mathrm{s}=0}^{3 \mathrm{k}+2} \mathrm{~S}_{4(\mathrm{p}+3 \mathrm{k})+12-4 \mathrm{~s}}=0 \\
& \mu_{4 \mathrm{p}+4}-\mathrm{x}_{3 \mathrm{p}+4} \prod_{\mathrm{s}=0}^{\mathrm{k}} \mathrm{S}_{4(\mathrm{p}+\mathrm{k})+5-4 \mathrm{~s}} \prod_{\mathrm{s}=0}^{2 \mathrm{k}+1} \mathrm{~S}_{4(\mathrm{p}+2 \mathrm{k})+9-4 \mathrm{~s}} \prod_{\mathrm{s}=0}^{3 \mathrm{k}+2} \mathrm{~S}_{4(\mathrm{p}+3 \mathrm{k})+13-4 \mathrm{~s}}=0
\end{aligned}
$$




$$
\mu_{4 \mathrm{p}+5}-\mathrm{x}_{3 \mathrm{p}+5}^{4} \prod_{\mathrm{s}=0}^{\mathrm{k}} \mathrm{S}_{4(\mathrm{p}+\mathrm{k})+6-4 \mathrm{~s}} \prod_{\mathrm{s}=0}^{2 \mathrm{k}+1} \mathrm{~S}_{4(\mathrm{p}+2 \mathrm{k})+10-4 \mathrm{~s}} \prod_{\mathrm{s}=0}^{3 \mathrm{k}+2} \mathrm{~S}_{4(\mathrm{p}+3 \mathrm{k})+14-4 \mathrm{~s}}=0
$$

Using the fact that $\sum_{\sigma} x_{i}(\sigma)=1$ for $i=4 p+2,4 p+3,4 p+4$ and $4 p+5$ from the previous equations one obtains

$$
\begin{gathered}
\lambda_{4 \mathrm{p}} \frac{\prod_{\mathrm{s}=0}^{\mathrm{k}} \lambda_{4(\mathrm{p}+\mathrm{k})-4 \mathrm{~s}} \prod_{\mathrm{s}=0}^{\mathrm{k}+1} \lambda_{4(\mathrm{p}+2 \mathrm{k})+4-4 \mathrm{~s}} \prod_{\mathrm{s}=0}^{\mathrm{k}+2} \lambda_{4(\mathrm{p}+3 \mathrm{k})+8-4 \mathrm{~s}}}{\prod_{\mathrm{s}=0}^{\mathrm{k}} \lambda_{4(\mathrm{p}+\mathrm{k})+1-4 \mathrm{~s}} \prod_{\mathrm{s}=0}^{2 \mathrm{k}+1} \lambda_{4(\mathrm{p}+2 \mathrm{k})+5-4 \mathrm{~s}} \prod_{\mathrm{s}=0}^{2 \mathrm{k}+2} \lambda_{4(\mathrm{p}+3 \mathrm{k})+9-4 \mathrm{~s}}}=\mathrm{b}_{3 \mathrm{p}} \\
\lambda_{4 \mathrm{p}+1} \frac{\prod_{\mathrm{s}=0}^{\mathrm{k}} \lambda_{4(\mathrm{p}+\mathrm{k})+1-4 \mathrm{~s}} \prod_{\mathrm{s}=0}^{2 \mathrm{k}+1} \lambda_{4(\mathrm{p}+2 \mathrm{k})+5-4 \mathrm{~s}} \prod_{\mathrm{s}=0}^{3 \mathrm{k}+2} \lambda_{4(\mathrm{p}+3 \mathrm{k})+9-4 \mathrm{~s}}}{\prod_{\mathrm{s}=0}^{\mathrm{k}} \lambda_{4(\mathrm{p}+\mathrm{k})+2-4 \mathrm{~s}} \prod_{\mathrm{s}=0}^{2 \mathrm{k}+1} \lambda_{4(\mathrm{p}+2 \mathrm{k})+6-4 \mathrm{~s}} \prod_{\mathrm{s}=0}^{3 \mathrm{k}+2} \lambda_{4(\mathrm{p}+3 \mathrm{k})+10-4 \mathrm{~s}}}=\mathrm{b}_{3 \mathrm{p}+1} \\
\lambda_{4 \mathrm{p}+2} \frac{\prod_{\mathrm{s}=0}^{\mathrm{k}} \lambda_{4(\mathrm{p}+\mathrm{k})+2-4 \mathrm{~s}} \prod_{\mathrm{s}=0}^{\mathrm{k}+1} \lambda_{4(\mathrm{p}+2 \mathrm{k})+6-4 \mathrm{~s}} \prod_{\mathrm{s}=0}^{2 \mathrm{k}+2} \lambda_{4(\mathrm{p}+3 \mathrm{k})+10-4 \mathrm{~s}}}{\prod_{\mathrm{s}=0}^{\mathrm{k}} \lambda_{4(\mathrm{p}+\mathrm{k})+3-4 \mathrm{~s}} \prod_{\mathrm{s}=0}^{\mathrm{k}+1} \lambda_{4(\mathrm{p}+2 \mathrm{k})+7-4 \mathrm{~s}} \prod_{\mathrm{s}=0}^{2 \mathrm{k}+2} \lambda_{4(\mathrm{p}+3 \mathrm{k})+11-4 \mathrm{~s}}}=\mathrm{b}_{3 \mathrm{p}+2} \\
\lambda_{4 \mathrm{p}+3} \frac{\prod_{\mathrm{s}=0}^{\mathrm{k}} \lambda_{4(\mathrm{p}+\mathrm{k})+3-4 \mathrm{~s}} \prod_{\mathrm{s}=0}^{2 \mathrm{k}+1} \lambda_{4(\mathrm{p}+2 \mathrm{k})+7-4 \mathrm{~s}} \prod_{\mathrm{s}=0}^{2 \mathrm{k}+2} \lambda_{4(\mathrm{p}+3 \mathrm{k})+11-4 \mathrm{~s}}}{\prod_{\mathrm{s}=0}^{\mathrm{k}} \lambda_{4(\mathrm{p}+\mathrm{k})+4-4 \mathrm{~s}} \prod_{\mathrm{s}=0}^{2 \mathrm{k}+1} \lambda_{4(\mathrm{p}+2 \mathrm{k})+8-4 \mathrm{~s}} \prod_{\mathrm{s}=0}^{2 \mathrm{k}+2} \lambda_{4(\mathrm{p}+3 \mathrm{k})+12-4 \mathrm{~s}}}=\mathrm{b}_{3 \mathrm{p}+3}
\end{gathered}
$$

where

$$
\begin{aligned}
& \mathrm{b}_{3 \mathrm{p}} \\
& =\left(\sum_{\sigma}\left(\frac{1}{\mathrm{a}_{4 \mathrm{p}}(\sigma)} \frac{\prod_{\mathrm{s}=0}^{\mathrm{k}} \mathrm{a}_{4(\mathrm{p}+\mathrm{k})+1-4 \mathrm{~s}}(\sigma) \prod_{\mathrm{s}=0}^{2 \mathrm{k}+1} \mathrm{a}_{4(\mathrm{p}+2 \mathrm{k})+5-4 \mathrm{~s}}(\sigma) \prod_{\mathrm{s}=0}^{\mathrm{k}+2} \mathrm{a}_{4(\mathrm{p}+3 \mathrm{k})+9-4 \mathrm{~s}}(\sigma)}{\prod_{4(\mathrm{p}+\mathrm{k})-4 \mathrm{~s}}(\sigma) \prod_{\mathrm{s}=0}^{2 \mathrm{k}+1} \mathrm{a}_{4(\mathrm{p}+2 \mathrm{k})+4-4 \mathrm{~s}}(\sigma) \prod_{\mathrm{s}=0}^{3 \mathrm{k}+2} \mathrm{a}_{4(\mathrm{p}+3 \mathrm{k})+8-4 \mathrm{~s}}(\sigma)}\right)^{4}\right)^{1 / 4}
\end{aligned}
$$

and $b_{3 p+1}, b_{3 p+2}, b_{3 p+3}$ are similar expression.

Now multiplying the equations (30) through (33) it appears

$$
\begin{gathered}
\mathrm{b}_{4 \mathrm{p}} \mathrm{b}_{4 \mathrm{p}+1} \mathrm{~b}_{4 \mathrm{p}+2} \mathrm{~b}_{4 \mathrm{p}+3}=\lambda_{4 \mathrm{p}} \lambda_{4 \mathrm{p}+1} \lambda_{4 \mathrm{p}+2} \lambda_{4 \mathrm{p}+3} . \\
\cdot \frac{\lambda_{4(\mathrm{p}+\mathrm{k})} \lambda_{4(\mathrm{p}+\mathrm{k})-4} \cdots \lambda_{4 \mathrm{p}+4} \lambda_{4 \mathrm{p}}}{\lambda_{4(\mathrm{p}+\mathrm{k})+4} \lambda_{4(\mathrm{p}+\mathrm{k})} \cdots \lambda_{4 \mathrm{p}+8} \lambda_{4 \mathrm{p}+4}} \\
\cdot \frac{\lambda_{4(\mathrm{p}+2 \mathrm{k})+4} \lambda_{4(\mathrm{p}+2 \mathrm{k})} \cdots \lambda_{4 \mathrm{p}+4} \lambda_{4 \mathrm{p}}}{\lambda_{4(\mathrm{p}+2 \mathrm{k})+8} \lambda_{4(\mathrm{p}+\mathrm{k})+4} \cdots \lambda_{4 \mathrm{p}+4}} \\
\cdot \frac{\lambda_{4(\mathrm{p}+3 \mathrm{k})+8} \lambda_{4(\mathrm{p}+3 \mathrm{k})+4} \cdots \lambda_{4 \mathrm{p}+4} \lambda_{4 \mathrm{p}}}{\lambda_{4(\mathrm{p}+3 \mathrm{k})+12} \lambda_{4(\mathrm{p}+3 \mathrm{k})+8} \cdots \lambda_{4 \mathrm{p}+4}}
\end{gathered}
$$




$$
=\lambda_{4 \mathrm{p}}^{4} \frac{\lambda_{4 \mathrm{p}+1} \lambda_{4 \mathrm{p}+2} \lambda_{4 \mathrm{p}+3}}{\lambda_{4(\mathrm{p}+\mathrm{k})+4} \lambda_{4(\mathrm{p}+2 \mathrm{k})+8} \lambda_{4(\mathrm{p}+3 \mathrm{k})+12}}
$$

but remembering that

$$
\begin{gathered}
4 p+1+4 k+3=4(p+k)+4 \\
4 p+2+8 k+6=4(p+2 k)+8 \\
4 p+3+12 k+9=4(p+3 k)+12
\end{gathered}
$$

then

$$
\lambda_{4 p}=\left(b_{4 p} b_{4 p+1} b_{4 p+2} b_{4 p+3}\right)^{1 / 4}
$$

In a similar way it is possible to obtain

$$
\lambda_{i}=\left(b_{i} b_{i+1} b_{i+2} b_{i+3}\right)^{1 / 4}
$$

Thus we have computed explicitly the $E$-point completely mixed. The value of $\mathrm{x}_{\mathrm{i}}(\sigma)$ are derived from the equations (25) and similar ones. It is clear that such $E$-point is the only one completely mixed for the diagonal game.

\section{A general game with $s k+1$ players with $1 \leq s \leq k$}

Now in this last section we are going to generalize the previous results obtained in the first part of the section 2 . Here we consider a game with sk +1 players with $1 \leq \mathrm{s} \leq \mathrm{k}$. The structure function is given by $\mathrm{d}(\mathrm{i})=\mathrm{N}-\{\mathrm{i}+2, \mathrm{i}+3, \mathrm{i}+4, \mathrm{i}+5, \ldots, \mathrm{i}+\mathrm{s}\}$ $\bmod \mathrm{sk}+1$.

The payoff functions are given by

$$
\mathrm{A}_{\mathrm{i}}\left(\sigma_{1} \ldots \sigma_{\mathrm{sk}+1}\right)=\mathrm{a}_{\mathrm{i}}\left(\sigma_{\mathrm{i}}\right) \delta\left(\sigma_{\mathrm{i}}, \sigma_{\mathrm{i}+2}, \sigma_{\mathrm{i}+3}, \ldots, \sigma_{\mathrm{i}+\mathrm{s}}\right), \quad \mathrm{a}_{\mathrm{i}}\left(\sigma_{\mathrm{i}}\right)>0 .
$$

The corresponding equations (4) for this game with the same notation as in the previous section is

$$
\mathrm{x}_{\mathrm{i}+\mathrm{s}}=\prod_{\mathrm{t}=0}^{\mathrm{r}} \mathrm{S}_{\mathrm{i}+1-\mathrm{ts}} \mathrm{x}_{\mathrm{i}-\mathrm{st}}
$$

For $\mathrm{i}=\mathrm{s}(\mathrm{p}+\mathrm{k})+1$ and $\mathrm{r}=\mathrm{k}$ it is obtained

$$
\mathrm{x}_{\mathrm{sp}+\mathrm{s}}=\prod_{\mathrm{t}=0}^{\mathrm{k}} \mathrm{S}_{\mathrm{s}(\mathrm{p}+\mathrm{k})+2-\mathrm{ts}} \mathrm{x}_{\mathrm{sp}+1}
$$

and similarly it is possible to derive 


$$
\mathrm{x}_{\mathrm{sp}+\mathrm{s}-1}=\prod_{\mathrm{t}=0}^{2 \mathrm{k}} \mathrm{S}_{\mathrm{s}(\mathrm{p}+2 \mathrm{k})+2-\mathrm{ts}} \mathrm{x}_{\mathrm{sp}+1}
$$

and

$$
\mathrm{x}_{\mathrm{sp}+\mathrm{s}-\mathrm{u}}=\prod_{\mathrm{t}=0}^{(\mathrm{u}+1) \mathrm{k}} \mathrm{S}_{\mathrm{s}(\mathrm{p}+(\mathrm{u}+1)) \mathrm{k}+2-\mathrm{ts}} \mathrm{x}_{\mathrm{sp}+1}
$$

Replacing in the adequate equation we get

$$
\mu_{\mathrm{sp}+1}-\mathrm{x}_{\mathrm{sp}+1}^{\mathrm{s}} \prod_{\mathrm{u}=0}^{\mathrm{s}-2} \prod_{\mathrm{t}=0}^{\mathrm{u}+1) \mathrm{k}} \mathrm{S}_{\mathrm{s}(\mathrm{p}+(\mathrm{u}+1) \mathrm{k})+2-\mathrm{ts}}=0
$$

or

$$
\mathrm{x}_{\mathrm{sp}+1}^{\mathrm{s}}=\mu_{\mathrm{sp}+1} \frac{\prod_{\mathrm{u}=0}^{\mathrm{s}-2} \prod_{\mathrm{t}=0}^{\mathrm{u}+1) \mathrm{k}} \mu_{\mathrm{s}(\mathrm{p}+(\mathrm{u}+1) \mathrm{k})+1-\mathrm{ts}}}{\prod_{\mathrm{u}=0}^{\mathrm{s}-2} \prod_{\mathrm{t}=0}^{\mathrm{u}+1) \mathrm{k}} \mu_{\mathrm{s}(\mathrm{p}+(\mathrm{u}+1) \mathrm{k})+2-\mathrm{ts}}}
$$

and from here

$$
\lambda_{\mathrm{sp}-1}=\frac{\prod_{\mathrm{u}=0}^{\mathrm{s}-2} \prod_{\mathrm{t}=0}^{\mathrm{u}+1) \mathrm{k}} \lambda_{\mathrm{s}(\mathrm{p}+(\mathrm{u}+1) \mathrm{k})-1-\mathrm{ts}}}{\prod_{\mathrm{u}=0}^{\mathrm{s}-2} \prod_{\mathrm{t}=0}^{\mathrm{u}+1) \mathrm{k}} \lambda_{\mathrm{s}(\mathrm{p}+(\mathrm{u}+1) \mathrm{k})-\mathrm{ts}}}=\mathrm{b}_{\mathrm{sp}+1}
$$

where

$$
\mathrm{b}_{\mathrm{sp}+1}=\left[\sum_{\sigma}\left(\frac{1}{\mathrm{a}_{\mathrm{sp}-1}(\sigma)} \frac{\prod_{\mathrm{u}=0}^{\mathrm{s}-2} \prod_{\mathrm{t}=0}^{\mathrm{u}+1) \mathrm{k}} \mathrm{a}_{\mathrm{s}(\mathrm{p}+(\mathrm{u}+1) \mathrm{k})-\mathrm{ts}}(\sigma)}{\prod_{\mathrm{u}=0}^{\mathrm{s}-2} \prod_{\mathrm{t}=0}^{\mathrm{u}+1) \mathrm{k}} \mathrm{a}_{\mathrm{s}(\mathrm{p}+(\mathrm{u}+1) \mathrm{k})-1-\mathrm{ts}}(\sigma)}\right)^{1 / \mathrm{s}}\right]^{\mathrm{s}}
$$

Similarly it is possible to derive

$$
\lambda_{\mathrm{sp}-1+\mathrm{q}} \frac{\prod_{\mathrm{u}=0}^{\mathrm{s}-2} \prod_{\mathrm{t}=0}^{\mathrm{u}+1) \mathrm{k}} \lambda_{\mathrm{s}(\mathrm{p}+(\mathrm{u}+1) \mathrm{k})-1+\mathrm{q}-\mathrm{ts}}}{\prod_{\mathrm{u}=0}^{\mathrm{s}-2} \prod_{\mathrm{t}=0}^{(\mathrm{u}+1) \mathrm{k}} \lambda_{\mathrm{s}(\mathrm{p}+(\mathrm{u}+1) \mathrm{k})+\mathrm{q}-\mathrm{ts}}}=\mathrm{b}_{\mathrm{sp}+1+\mathrm{q}}
$$

where the values $b_{s p+1+q}$ might be obtained in a similar way as $b_{s p+1}$ in (45). Multiplying the b's we get

$$
\prod_{\mathrm{q}=1}^{\mathrm{s}} \mathrm{b}_{\mathrm{sp}+\mathrm{q}}=\prod_{\mathrm{q}=1}^{\mathrm{s}} \lambda_{\mathrm{sp}+\mathrm{q}-2} \frac{\prod_{\mathrm{u}=0}^{\mathrm{s}-2} \prod_{\mathrm{t}=0}^{\mathrm{u}+1) \mathrm{k}} \lambda_{\mathrm{s}(\mathrm{p}+(\mathrm{u}+1) \mathrm{k})-2+\mathrm{q}-\mathrm{ts}}}{\prod_{\mathrm{u}=0}^{\mathrm{s}-2} \prod_{\mathrm{t}=0}^{\mathrm{u}+1) \mathrm{k}} \lambda_{\mathrm{s}(\mathrm{p}+(\mathrm{u}+1) \mathrm{k})-1+\mathrm{q}-\mathrm{ts}}}
$$

or

$$
\prod_{\mathrm{q}=1}^{\mathrm{s}} \mathrm{b}_{\mathrm{sp}+\mathrm{q}}=\prod_{\mathrm{q}=1}^{\mathrm{s}} \lambda_{\mathrm{sp}+\mathrm{q}-2} \frac{\prod_{\mathrm{u}=0}^{\mathrm{s}-2} \prod_{\mathrm{t}=0}^{\mathrm{u}+1) \mathrm{k}} \lambda_{\mathrm{s}(\mathrm{p}+(\mathrm{u}+1) \mathrm{k})-1-\mathrm{ts}}}{\prod_{\mathrm{u}=0}^{\mathrm{s}-2} \prod_{\mathrm{t}=0}^{(\mathrm{u}+1) \mathrm{k}} \lambda_{\mathrm{s}(\mathrm{p}+(\mathrm{u}+1) \mathrm{k})-1+\mathrm{s}-\mathrm{ts}}} .
$$

In (48) we can consider a term for fixed $u$, then we have 


$$
\begin{gathered}
\frac{\prod_{\mathrm{t}=0}^{\mathrm{u}+1) \mathrm{k}} \lambda_{\mathrm{s}(\mathrm{p}+(\mathrm{u}+1) \mathrm{k})-1-\mathrm{ts}}}{\prod_{\mathrm{t}=0}^{\mathrm{u}+1) \mathrm{k}} \lambda_{\mathrm{s}(\mathrm{p}+(\mathrm{u}+1) \mathrm{k})-1+\mathrm{s}-\mathrm{ts}}}=\frac{\lambda_{\mathrm{s}(\mathrm{p}+(\mathrm{u}+1) \mathrm{k})-1} \lambda_{\mathrm{s}(\mathrm{p}+(\mathrm{u}+1) \mathrm{k})-1-\mathrm{s}} \cdots}{\lambda_{\mathrm{s}(\mathrm{p}+(\mathrm{u}+1) \mathrm{k})-1+\mathrm{s}} \lambda_{\mathrm{s}(\mathrm{p}+(\mathrm{u}+1) \mathrm{k})-1}} \\
\frac{\cdots \lambda_{\mathrm{sp}-1+\mathrm{s}} \lambda_{\mathrm{sp}-1}}{\lambda_{\mathrm{sp}-1+\mathrm{s}}}=\frac{\lambda_{\mathrm{sp}-1}}{\lambda_{\mathrm{s}(\mathrm{p}+(\mathrm{u}+1) \mathrm{k})-1-\mathrm{s}}}
\end{gathered}
$$

therefore

$$
\begin{gathered}
\prod_{\mathrm{q}=1}^{\mathrm{s}} \mathrm{b}_{\mathrm{sp}+\mathrm{q}}=\prod_{\mathrm{q}=1}^{\mathrm{s}} \lambda_{\mathrm{sp}+\mathrm{q}-2} \prod_{\mathrm{u}=0}^{\mathrm{s}-2} \frac{\lambda_{\mathrm{sp}-1}}{\lambda_{\mathrm{s}(\mathrm{p}+(\mathrm{u}+1) \mathrm{k})-1+\mathrm{s}}} \\
=\lambda_{\mathrm{sp}-1}^{\mathrm{s}} \frac{\lambda_{\mathrm{sp}} \lambda_{\mathrm{sp}+1} \lambda_{\mathrm{sp}+2} \cdots \lambda_{\mathrm{sp}+\mathrm{s}-3} \lambda_{\mathrm{sp}+\mathrm{s}-2}}{\lambda_{\mathrm{s}(\mathrm{p}+\mathrm{k})-1+\mathrm{s}} \lambda_{\mathrm{s}(\mathrm{p}+2 \mathrm{k})-1+\mathrm{s}} \cdots \lambda_{\mathrm{s}(\mathrm{p}+(\mathrm{s}-1) \mathrm{k})-1+\mathrm{s}}}
\end{gathered}
$$

but remembering that

$$
\begin{aligned}
& \mathrm{sp}+\mathrm{s}-2+\mathrm{sk}+1=\mathrm{s}(\mathrm{p}+\mathrm{k})-1+\mathrm{s} \\
& \mathrm{sp}+\mathrm{s}-3+2 \mathrm{sk}+2=\mathrm{s}(\mathrm{p}+2 \mathrm{k})-1+\mathrm{s} \\
& \mathrm{sp}+(\mathrm{s}-1) \mathrm{sk}+\mathrm{s}-1=\mathrm{s}(\mathrm{p}+(\mathrm{s}-1) \mathrm{k})-1+\mathrm{s}
\end{aligned}
$$

the (50) takes the form

$$
\lambda_{\mathrm{sp}-1}^{\mathrm{s}}=\prod_{\mathrm{q}=1}^{\mathrm{s}} \mathrm{b}_{\mathrm{sp}+\mathrm{q}}
$$

or

$$
\lambda_{\mathrm{sp}-1}=\left(\prod_{\mathrm{q}=1}^{\mathrm{s}} \mathrm{b}_{\mathrm{sp}+\mathrm{q}}\right)^{1 / \mathrm{s}}
$$

thus we have computed explicitly the completely mixed $E$-point in the game $(\Gamma, E)$.

As a final remark we would like to say that with the same technique it would be possible to compute the $E$-points in the case that we have $\mathrm{sk}+\overline{\mathrm{s}}$ instead of $\mathrm{sk}+1$ with the property

$$
\left\{\mathrm{sk}+\overline{\mathrm{s}}, 2 \mathrm{~s}+\mathrm{k}+2 \overline{\mathrm{s}}, 3 \mathrm{sk}+3 \overline{\mathrm{s}}, \ldots, \mathrm{s}^{2} \mathrm{k}+\mathrm{s} \mathrm{s}\right\}=\{1,2, \ldots, \mathrm{s}\}
$$

If this last condition is not satisfied then the problem of the existence and the computation of $E$-point becomes complex. 


\section{References}

(1945) Kaplansky I.: A Contribution of von Neumann's Theory of Games. Annals of Mathematics Vol. 46 pp. 474-479.

(1967) Marchi, E.: E-points for Games. Proc. Mat. Acad. of Sciences U.S.A. Vol. 57 $\mathrm{N}^{\circ} 4$ pp. 878-882.

(1990) -: On Equilibrium Points of Diagonal N-Person Games. JOTA Vol. 64, №1.

(2004) -: E-points of Diagonal Games I, II (to be published)

(1989) Martínez, R.: Cálculo de E-points en juegos tripersonales. Doctoral Thesis Universidad Nacional de San Luis, San Luis, Argentina, 1989. 